\title{
Field effect on positron diffusion in semi-insulating GaAs
}

\author{
Y. Y. Shan, P. Asoka-Kumar, and K. G. Lynn \\ Department of Physics, Brookhaven National Laboratory, Upton, New York 11793
}

S. Fung and C. D. Beling

Department of Physics, The University of Hong Kong, Hong Kong

(Received 28 February 1996)

\begin{abstract}
An energy-tunable monoenergetic positron beam was used to study positron diffusion in the space-charge region of an $\mathrm{Au} / \mathrm{GaAs}(\mathrm{SI})$ (semi-insulating) Schottky contact, where the electric field reaches $\sim 10^{5} \mathrm{~V} \mathrm{~cm}{ }^{-1}$ by reverse biasing the diode. An analytical solution of the time-dependent positron drift-diffusion model under an electric field was obtained for the case of a semi-infinite body with a capturing boundary, and explains the experimental results well. A positron diffusion coefficient of $1.8 \pm 0.2 \mathrm{~cm}^{2} \mathrm{~s}^{-1}$, and a positron mobility of $70 \pm 10 \mathrm{~cm}^{2} \mathrm{~V}^{-1} \mathrm{~s}^{-1}$ in $\mathrm{GaAs}(\mathrm{SI})$ at $300 \mathrm{~K}$, were obtained independently. This result is consistent with the Einstein relation. The dependence of the positron current density at the Au/GaAs interface on the electric field shows that $\mathrm{GaAs}(\mathrm{SI})$ is a possible candidate for the fabrication of the field-assisted positron moderator. [S0163-1829(96)04428-1]
\end{abstract}

\section{INTRODUCTION}

Studies of carrier transport in solids provide unique information about mechanisms of interactions in the material. Positrons exhibit transport properties similar to those of electrons and holes. The motion of positrons is of interest for understanding the mechanisms of positron scattering, and for various applications of the positron annihilation technique in condensed matter.

Positron diffusion in several cubic metals was studied extensively by Soininen et al., ${ }^{1}$ and the diffusion coefficient is $1-2 \mathrm{~cm}^{2} \mathrm{~s}^{-1}$ at $300 \mathrm{~K}$. Mills and co-workers studied positron motion in semiconductors by measuring drift velocities, based on the Doppler shift in the annihilation radiation in $\mathrm{Ge}$ and Si crystals of $\gamma$ detectors. ${ }^{2,3}$ They showed that phonon scattering is the main lattice-scattering mechanism for positrons. A similar experiment was carried out in GaAs, and a low positron mobility of $20 \mathrm{~cm}^{2} \mathrm{~V}^{-1} \mathrm{~s}^{-1}$ was reported at $300 \mathrm{~K}^{4}$

Simpson et al. $^{5}$ measured positron mobility in Si using the positron-lifetime technique. ${ }^{5}$ Recently, a positron mobility value of $60 \mathrm{~cm}^{2} \mathrm{~V}^{-1} \mathrm{~s}^{-1}$ in GaAs at $300 \mathrm{~K}$ was reported using the lifetime method, in which the effect of the strong electric field inside the space-charge region was considered. ${ }^{6}$

Measurements of positron diffusion using monoenergetic positron beams were discussed in detail by Schultz and Lynn $^{7}$ and Huomo, Soininen, and Vehanen. ${ }^{8}$ Positron diffusion coefficients in $\mathrm{Si}$ and $\mathrm{Ge}$ obtained in early slow-beam experiments varied significantly. ${ }^{9-14}$ Space-charge effects at semiconductor surfaces were suggested to explain some of these anomalies. ${ }^{9,12,15}$ The diffusion coefficient of $3.0 \pm 0.25$ $\mathrm{cm}^{2} \mathrm{~s}^{-1}$ in $n$-type $\mathrm{Si}$ was obtained by numerically solving the quasistationary drift-diffusion equation by considering the effect of the electric field in the space-charge region. ${ }^{16}$ In GaAs, diffusion lengths of $1800 \pm 140$ (Ref. 17) and $1400 \AA$ (Ref. 18) were reported for $n$-type GaAs at $300 \mathrm{~K}$, which correspond to positron diffusion coefficients of $0.9-1.4$ $\mathrm{cm}^{2} \mathrm{~s}^{-1}$. A diffusion coefficient of $1.6(2) \mathrm{cm}^{2} \mathrm{~s}^{-1}$ in
GaAs(SI) (semi-insulating) was reported recently. ${ }^{19}$ All these results were obtained by numerically fitting the diffusion model without including the electric field.

The diffusion-equation approach is used frequently in analyzing the results from positron beam experiments. ${ }^{20}$ To model slow-positron beam experiments and obtain the positron current density, the time-dependent diffusion equation has to be used. ${ }^{21-24}$

In this work, we present results of a positron beam study in semi-insulating (SI) GaAs under large reverse bias $\left(10^{5}\right.$ $\left.\mathrm{V} \mathrm{cm}{ }^{-1}\right)$. It is similar to the method used elsewhere. ${ }^{16,25}$ The time-dependent drift-diffusion equation with a capturing boundary in semi-infinite space and electric field can be solved analytically. It shows that the positron diffusion coefficient and positron mobility can be experimentally determined without implementing the Nernst-Einstein relation, thus providing a consistency check for the experimental numbers. We also discuss the relationship between the maximum fraction of the positron drift to the interface, the external bias, and the implantation energy.

\section{EXPERIMENT}

The sample used was undoped liquid-encapsulated Czochralski-grown SI GaAs(100) with a room-temperature resistivity of $10^{8} \Omega \mathrm{cm}$ and thickness of $0.5 \mathrm{~mm}$, and was purchased from ICI Wafer Technology Ltd. The EL2 concentration of the wafer was given as $1.5 \times 10^{16} \mathrm{~cm}^{-3}$. The substrate was annealed at $500{ }^{\circ} \mathrm{C}$ under forming gas $(80 \%$ $\mathrm{N}_{2}$ and $20 \% \mathrm{H}_{2}$ ) for $\mathrm{h}$ to reduce possible positron trapping defects inside the bulk. The sample then was degreased in acetone and ethanol before being etched in standard $\mathrm{NH}_{4} \mathrm{OH}: \mathrm{H}_{2} \mathrm{O}_{2}: \mathrm{H}_{2} \mathrm{O}(3: 1: 90)$ and $\mathrm{H}_{2} \mathrm{SO}_{4}: \mathrm{H}_{2} \mathrm{O}_{2}: \mathrm{H}_{2}$ $\mathrm{O}(8: 1: 1)$ solutions for $1 \mathrm{~min}$. A $1000-\AA$ gold layer was evaporated onto each side of the substrate's surface, forming a circular spot $8 \mathrm{~mm}$ in diameter. A dc bias was applied across the sample so that the internal electric field was opposite to the direction of beam injection. The $I-V$ character- 
istics of the sample were recorded using a picoammeter and a digital voltmeter.

Positron annihilation spectroscopy measurements were carried out with a magnetically guided positron beam at Brookhaven National Laboratory. Fast positrons from a $80-\mathrm{m} \mathrm{Ci}{ }^{22} \mathrm{Na}$ source were moderated in a $1-\mu \mathrm{m}$-thick single-crystal W(100) foil in transmission geometry. The intensity of the slow positron beam was about $3 \times 10^{5} e^{+} / \mathrm{s}$, and its diameter was $4 \mathrm{~mm}$. The incident-beam energy was varied from 0.1 to $50 \mathrm{keV}$. The pressure was $10^{-7}$ mbar. The 511-keV annihilation $\gamma$ spectra were detected and accumulated by a high-purity Ge detector and a digitally stabilized multichannel analyzer. A total of $1 \times 10^{6}$ counts were collected under the annihilation photopeak for each positron energy.

\section{POSITRON IMPLANTATION AND TIME-DEPENDENT DRIFT-DIFFUSION MODEL}

As a low monoenergetic positron beam is implanted into solids, positrons thermalize very rapidly, within $10 \mathrm{ps}^{7}$ The positron stopping profile $P_{E}(z)$ can be approximately described as a Makhovian distribution. ${ }^{26}$ Based on the Monte Carlo simulations of slowing down, and the experimental studies of multilayer structures, the stopping profile is taken as $^{27-30}$

$$
P(z)=m \frac{z^{m-1}}{z_{0}^{m}} \exp \left[-\left(\frac{z+a}{z_{0}}\right)^{m}\right],
$$

where $a=\left(\rho_{\mathrm{Au}} / \rho_{\mathrm{GaAs}}\right) \Delta$, and $\rho_{\mathrm{Au}}$, and $\rho_{\mathrm{GaAs}}$ are densities (in $\mathrm{g} \mathrm{cm}^{-3}$ ) of the Au overlayer and the GaAs crystal, respectively. $\Delta$ is the thickness of the Au layer. $z$ denotes the distance from the interface along the direction of positron beam injection. The parameters are taken as $\alpha=450$ $\AA \mathrm{g} \mathrm{cm}^{-3}, m=2.0$, and $n=1.6 .{ }^{30-32}$ The mean implantation depth equals

$$
\bar{z}=z_{0} \Gamma\left(1+\frac{1}{m}\right)
$$

where $z_{0}=\left(\alpha / \rho_{\mathrm{GaAs}}\right) E^{n}$, and the beam energy $E$ is in $\mathrm{keV}$. The electric field is not expected to distort the stopping profile of the $\mathrm{keV}$ positrons.

Once thermalized, positrons undergo diffusive motion inside the sample. By using Fick's law and the continuity equation, positron evolution in one dimension can be described by the diffusion-annihilation equation ${ }^{33}$

$$
\frac{\partial n(z, t)}{\partial t}=D_{+} \frac{\partial^{2} n(z, t)}{\partial z^{2}}-\nu_{+} \frac{\partial n(z, t)}{\partial z}-\lambda n(z, t),
$$

where $n(z, t)$ is the positron density, $D_{+}$is the positron diffusion coefficient, $\nu_{+}$is the electric-field-dependent positron drift velocity, $\nu_{+}=\mu_{+} E$, and $\lambda$ is the positron annihilation rate. The positron current density is given by

$$
j(z, t)=-D_{+} \frac{\partial n(z, t)}{\partial z}+n(z, t) \nu_{+}
$$

The system can be treated as a semi-infinite system with a perfectly absorbing boundary at the interface. The perfect absorbing condition requires positron current density to van- ish at the interface. ${ }^{33,34}$ The equation can be solved under the initial condition of $n(z, 0)=P_{E}(z)$. The positron distribution is obtained as

$$
\begin{aligned}
n(z, t)= & \frac{e^{-\lambda t}}{2 \sqrt{\pi D_{+} t}} \int_{0}^{+\infty} P_{E}(\xi)\left[e^{-\left[\xi-\left(z-\nu_{+} t\right)\right]^{2} / 4 D_{+} t}\right. \\
& \left.-e^{\left(\nu_{+} / D_{+}\right) \xi} e^{-\left[\xi+\left(z-\nu_{+} t\right)\right]^{2} / 4 D_{+} t}\right] d \xi
\end{aligned}
$$

Using Eqs. (4) and (5), the fraction of positrons reaching the interface can be obtained as

$$
\begin{aligned}
F_{\text {in }} & =\left.\int_{0}^{\infty} j(z, t)\right|_{z=0} d t \\
& =\int_{0}^{\infty} P_{E}(\xi) e^{\left.-\left[\left(\nu_{+} / 2 D_{+}\right)+\sqrt{\left(\lambda / D_{+}\right)+\nu_{+} / 2 D_{+}}\right)^{2}\right] \xi} d \xi .
\end{aligned}
$$

When $j<0$ and $\nu_{+}<0$ mean the direction of the positron current density, and the internal electric field is toward the $-z$ direction, opposite to the direction of positron beam injection. Rewriting the variable $\xi$ to $z$, we obtain

$$
F_{\text {in }}=\int_{0}^{\infty} P_{E}(z) e^{-\left[\left(\nu_{+} / 2 D_{+}\right)+\sqrt{\left(\lambda / D_{+}\right)+\left(\nu_{+} / 2 D_{+}\right)^{2}}\right] z} d z .
$$

A different derivation of Eq. (7) was given by Mills and Murray. ${ }^{15}$ When the electric field is zero, Eq. (7) reduces to the results obtained in the previous works. ${ }^{17,18,28}$

Positron annihilation at various incident energies is measured by analyzing the Doppler-broadening spectrum of the 511-keV annihilation which reflects the momentum distribution of the annihilating electron-positron pair. The Dopplerbroadening parameter $S$ is defined as the ratio of the counts in the central part of the $511-\mathrm{keV}$ annihilation line to the total number of counts in the annihilation peak. The measured $S$ value is the linear combination of the $S$ parameter contributions from different annihilation states, ${ }^{35}$ i.e., $S(E)=\Sigma f_{i}(E) S_{i}$, where $f_{i}(E)$ is the fraction of positrons annihilating in the $i$ th state characterized by the $S_{i}$ parameter. The fractions $f_{i}(E)$ can be obtained by solving the diffusion equation, subject to the positron implantation profile and boundary conditions.

As in the previous work, ${ }^{36}$ the average electric field in the depletion region of the GaAs is taken as

$$
E=\frac{e N_{D}}{2 \varepsilon_{0} \varepsilon_{r}} W=\left[\frac{e N_{D}\left(\phi_{\mathrm{bi}}+V_{d}\right)}{2 \varepsilon_{0} \varepsilon_{r}}\right]^{1 / 2},
$$

where $W$ is the bias-dependent width of the depletion region, given by ${ }^{37}$

$$
W=\left[\frac{2 \varepsilon_{0} \varepsilon_{r}\left(\phi_{\mathrm{bi}}+V_{d}\right)}{e N_{D}}\right]^{1 / 2},
$$

$\varepsilon_{r}$ is the relative permittivity, and $N_{D}$ is the concentration of deep donor EL2. $\phi_{\mathrm{bi}}$ is the "built-in" contact potential given by $e \phi_{\mathrm{bi}}=\phi_{b}-\left(E_{c}-E_{f}\right)$, and $\phi_{b}$ is the Schottky barrier height. $E_{c}-E_{f}$ is the bulk Fermi level measured from the bottom of the conduction band. $V_{d}=V-I R_{b}$ is the voltage drop across the depletion region. $V$ is the applied bias, $R_{b}$ is the bulk resistance, and $I$ is the current flowing through the sample obtained from the $I-V$ measurement shown in Fig. 1. 


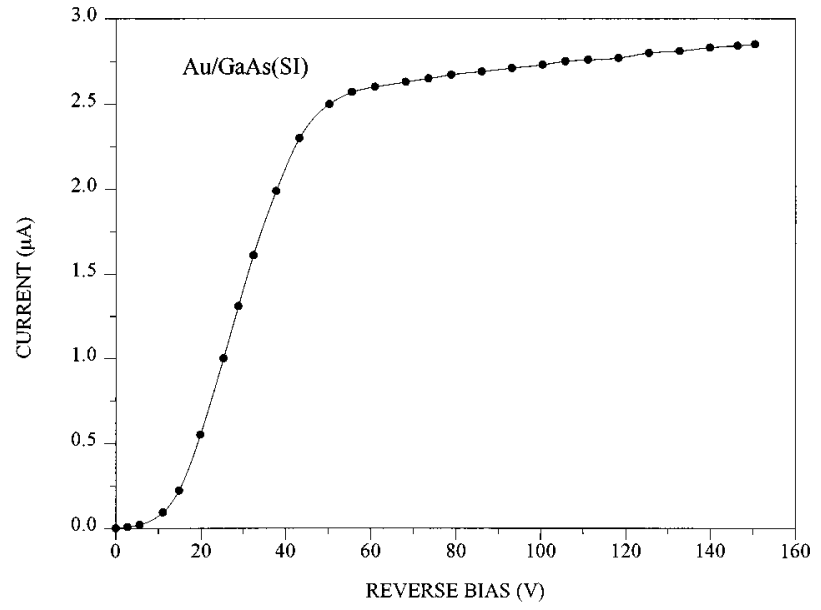

FIG. 1. The current-voltage characteristics of the $\mathrm{Au} / \mathrm{GaAs}(\mathrm{SI})$ sample under reverse bias.

\section{RESULTS AND DISCUSSIONS}

Figure 2 shows the $S$ parameter as a function of the incident positron beam energy under different biases. When the $\mathrm{Au} / \mathrm{GaAs}$ contact is reverse biased, the positrons are injected against the electric field, which aids their diffusion back to the interface. At very low implantation energies of less than $1 \mathrm{keV}$, an $S$ value of $0.4550-0.4600$ characterizes positrons annihilating mainly on the Au surface. As the energy increases, more positrons annihilate in the Au overlayer, and an $S$ value of $0.4490(5)$ is observed. That the $S-E$ valley or plateau characterizes the Au film was verified by the previous experiment where a positron slow beam study was carried out on $\mathrm{GaAs}$ with a series of $\mathrm{Au}$ overlayers with various thicknesses. ${ }^{38}$ It was observed that the thicker the $\mathrm{Au}$ film,

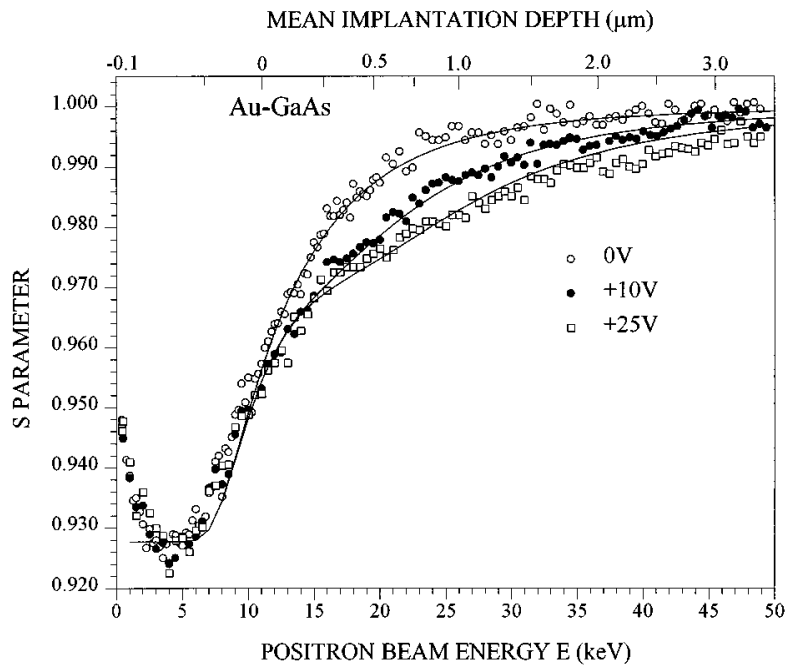

FIG. 2. The line-shape parameter $S$ as a function of the incident positron beam energy. Data for zero bias and reverse biases of 10 and $25 \mathrm{~V}$ are shown. The mean implantation depth for incident energy, $E<11 \mathrm{keV}$ and $E \geqslant 11 \mathrm{keV}$, represents positron stopping mainly in the $1000-\AA$-thick Au overlayer and in bulk GaAs, respectively. The solid lines are the drift-diffusion model calculations for $D_{+}=1.8 \mathrm{~cm}^{2} \mathrm{~s}^{-1}$ and $\mu_{+}=70 \mathrm{~cm}^{2} \mathrm{~V}^{-1} \mathrm{~s}^{-1}$.
TABLE I. The values of the parameters used in modeling the experimental data.

\begin{tabular}{lcc}
\hline \hline Density & $\rho_{\mathrm{Au}}$ & $19.3 \mathrm{~g} \mathrm{~cm}^{-3}$ \\
& $\rho_{\mathrm{GaAs}}$ & $5.32 \mathrm{~g} \mathrm{~cm}^{-3}$ \\
\hline Relative permittivity of GaAs & $\varepsilon_{r}$ & 13.2 \\
Sample resistance at $300 \mathrm{~K}$ & $R_{b}$ & $9.95 \times 10^{6} \Omega$ \\
Thickness of the Au layer & $\Delta$ & $1000 \AA$ \\
Deep donor concentration & $N_{D}$ & $1.5 \times 10^{16} \mathrm{~cm}^{-3}$ \\
Built-in potential & $\phi_{\mathrm{bi}}$ & $0.3 \mathrm{eV}$ \\
Implantation profile & $\alpha$ & $450 \AA \mathrm{g} \mathrm{cm}^{-3}$ \\
& $m$ & 2.0 \\
Positron annihilation rate & $n$ & 1.6 \\
\hline \hline
\end{tabular}

the wider the plateau. The calculation shows that $S$ parameter begins to increase from its plateau value when the beam energy is larger than $6 \mathrm{keV}$. Implanted positrons begin to penetrate through the Au layer into the interface and bulk when the implantation energy is over $6 \mathrm{keV}$. This result is in good agreement with the experimental data. As the beam energy increases further, the $S$ parameter rises to a saturation value of $0.4840(5)$ for the zero-biased spectrum. This value can be taken as the bulk value $S_{b}$, to which all measured $S$ values were normalized. Therefore, the normalized surface and Au film $S$ values are 0.945(5) and 0.927(1), respectively. After normalization, bulk GaAs will have an $S$ value of 1.0. For reverse-biased spectra, $S(E)$ decreases in the positron energy range of $15-35 \mathrm{keV}$ in which positrons are mainly implanted into the depletion region (with high electric field) and drift back to the interface. Since the $\mathrm{Au} / \mathrm{GaAs}$ interface region has a large concentration of open-volume defects, ${ }^{39}$ this interface can be approximated as a perfect absorbing boundary. Based on the drift-diffusion model discussed in Sec. III, the $S$ parameter can be modeled as

$$
S(E, V)=F_{\mathrm{Au}} S_{\mathrm{Au}}+\left(1-F_{\mathrm{Au}}\right)\left[F_{\mathrm{in}} S_{\mathrm{in}}+\left(1-F_{\mathrm{in}}\right) S_{b}\right],
$$

where $F_{\text {in }}$ is calculated from Eq. (7). Values of 0.9277 for $S_{\mathrm{Au}}$, and 1.0 for $S_{b}$, are taken. Table I lists the values of the constants used in the calculation. In Eq. (10), the contribution of Au surface annihilation, $F_{s} S_{s}$, is included in the $\mathrm{Au}$ overlayer contribution, $F_{\mathrm{Au}} S_{\mathrm{Au}}$, and will not affect the extraction of the positron diffusion character inside the GaAs. Hence the theoretical $S-E$ curve is flat (the solid line in Fig. 2) when the beam energy is lower than $6 \mathrm{keV}$. As was pointed out, ${ }^{39}$ the evaporated Au film is full of defects, and the positron lifetime in Au is relatively short; therefore, the implanted thermalized positrons in the Au overlayer are assumed to be trapped and annihilate inside the Au layer. $F_{\text {Au }}$ can be expressed approximately as

$$
F_{\mathrm{Au}}=\int_{-a}^{0} P_{E}(z) d z .
$$

The fitted results using the above model are shown as the solid lines in Fig. 2. The $S_{\text {in }}$ value of 0.973(1) in the interface is deduced. The discrepancy between the theory and experimental data in the range of $5-8 \mathrm{keV}$ comes from the uncer- 


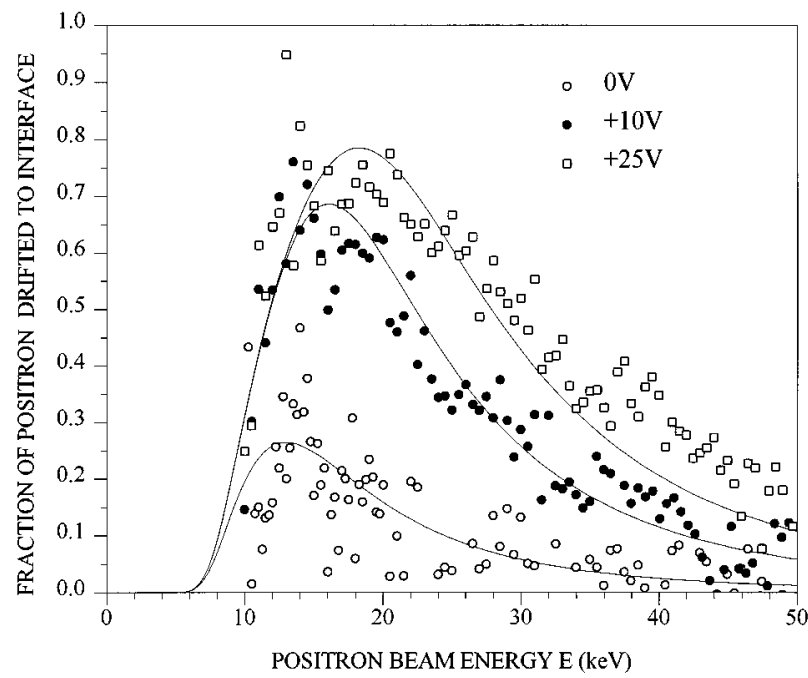

FIG. 3. The fraction of positrons drifting back to the interface for different biases and different incident-beam energies. The solid lines are calculated from Eq. (7), corresponding to $D_{+}=1.8 \pm 0.2$ $\mathrm{cm}^{2} \mathrm{~s}^{-1}$ and $\mu_{+}=70 \pm 10 \mathrm{~cm}^{2} \mathrm{~V}^{-1} \mathrm{~s}^{-1}$.

tainty of the Au film density (causing the evaporated layers to be less than the value of $19.3 \mathrm{~g} \mathrm{~cm}^{-3}$ used in the fitting). This variation in density will give rise to the uncertainty of the parameter $a$ involved in the implantation profile, Eq. (1). For the case of a high bias of $25 \mathrm{~V}$, the deviation of the fitted curve to the data points for positron energies above $30 \mathrm{keV}$ may arise from the approximations resorted to in the model. One is the linear approximation between the positron drift velocity and the high electric field experienced by positrons. The other is the average electric-field approximation used in the depletion region where, in fact, the electric field is not uniform according to the depletion model.

The determination of the positron diffusion coefficient $D_{+}$and positron mobility $\mu_{+}$was carried out as follows. First, consider the case when zero bias is applied to the sample; then the electric field is zero, and Eq. (7) becomes

$$
F_{\text {in }}=\int_{0}^{\infty} P_{E}(z) e^{-\sqrt{\left(\lambda / D_{+}\right)} z} d z
$$

Thus $F_{\text {in }}$ is a function of $D_{+}$. By fitting Eq. (10) to the zero-biased spectrum, a diffusion coefficient of $1.8 \pm 0.2$ $\mathrm{cm}^{2} \mathrm{~s}^{-1}$ is obtained. Then Eq. (10) is fitted to the different bias $S-E$ data, where $F_{\text {in }}$ is a function of $D_{+}$and $\mu_{+}$as expressed in Eq. (7). Using this $D_{+}$value, a positron mobility $\mu_{+}$of $70 \pm 10 \mathrm{~cm}^{2} \mathrm{~V}^{-1} \mathrm{~s}^{-1}$ is obtained. Unlike previous works where one of the coefficients was derived from the experimental data and the other obtained by using the Einstein relation, here both $D_{+}$and $\mu_{+}$are determined directly.

Figure 3 compares the theoretical $F_{\text {in }}$ value with the experimental value obtained by

$$
F_{\text {in }}=\frac{1}{S_{\text {in }}-S_{b}}\left[\frac{S(E, V)-F_{\mathrm{Au}} S_{\mathrm{Au}}}{1-F_{\mathrm{Au}}}-S_{b}\right] .
$$

Good agreement is found, except for beam energies less than $8 \mathrm{keV}$; this is because when the beam energy is low, the positrons stop predominantly in the Au overlayer, so the ex-

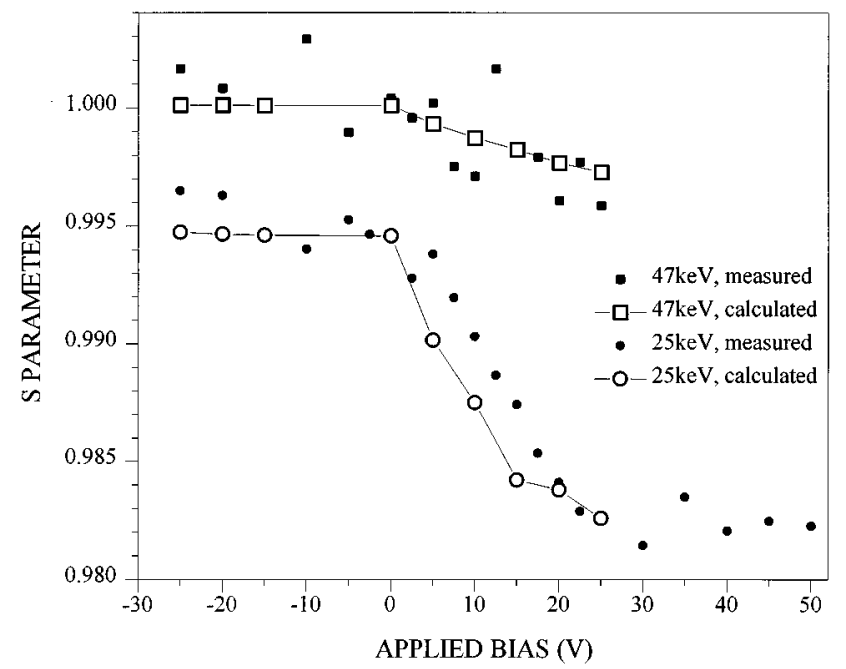

FIG. 4. The line-shape parameter $S$ as a function of the applied bias for incident positron energies of 25 and $47 \mathrm{keV}$. A positron diffusion coefficient of $1.8 \mathrm{~cm}^{2} \mathrm{~s}^{-1}$ and a positron mobility of 70 $\mathrm{cm}^{2} \mathrm{~V}^{-1} \mathrm{~s}^{-1}$ were used in the calculation.

perimental $F_{\text {in }}$ value is very small and shows a relatively large uncertainty. A maximum saturation $F_{\text {in }}$ value of about $75 \%$ was obtained for a beam energy of $18 \mathrm{keV}$, and a reverse bias of $25 \mathrm{~V}$. This result demonstrates that $F_{\text {in }}$ can be increased by properly biasing the sample, and, hence, that semi-insulating GaAs is a potential substrate material for making high-efficiency field-assisted positron moderators.

Figure 4 shows the $S$ parameter as a function of the applied bias measured at constant incident positron beam energies of 25 and $47 \mathrm{keV}$, corresponding to mean implantation depths of 0.94 and $3.2 \mu \mathrm{m}$, respectively. The electric-field effect of positron diffusion is apparent by the changes in the $S$ parameter. As the reverse bias increases (positive voltage values), the $S$ value decreases because a higher fraction of positrons drift to the $\mathrm{Au} / \mathrm{GaAs}$ interface. The $S$ parameter reaches a constant value when the reverse bias is larger than $25 \mathrm{~V}$, indicating a saturation of drift. The solid lines are the theoretical results with $D_{+}=1.8 \pm 0.2 \mathrm{~cm}^{2} \mathrm{~s}^{-1}$, $\mu_{+}=70 \pm 10 \mathrm{~cm}^{2} \mathrm{~V}^{-1} \mathrm{~s}^{-1}$, and $S_{\text {in }}=0.973(1)$. The gradient of the $S-V$ curve can be written as

$$
\frac{d S}{d V}=\left(1-F_{\mathrm{Au}}\right)\left(S_{\mathrm{in}}-S_{b}\right) \frac{d F_{\text {in }}}{d V},
$$

reflecting how effectively the electric field affects the positron transport. For reverse bias, $d S / d V<0$ when $S_{\text {in }}<S_{b}$, and $d S / d V>0$ when $S_{\text {in }}>S_{b}$.

\section{CONCLUSIONS}

A variable energy positron beam of $0.1-50 \mathrm{keV}$ was used to study positron diffusion in semi-insulating GaAs. The effect of the high electric field in the Au/GaAs depletion region on positron diffusion was observed. An analytical solution to the time-dependent positron diffusion model under an electric field in a semi-infinite body with a perfectly capturing boundary was derived which describes the experimental data well. Without resorting to the Einstein relationship, as in studies by others, a positron diffusion coefficient of 
$1.8 \pm 0.2 \mathrm{~cm}^{2} \mathrm{~s}^{-1}$ and positron mobility of $70 \pm 10$ $\mathrm{cm}^{2} \mathrm{~s}^{-1}$ in $\mathrm{GaAs}(\mathrm{SI})$ at $300 \mathrm{~K}$ were directly obtained from the experimental data. These results are consistent with the Einstein relation. The multilayer implantation profile $\mathrm{e}^{30}$ used here describes the present $\mathrm{Au} / \mathrm{GaAs}$ system reasonably well, but certainty about the layer densities, which may differ from their crystalline values, will generate errors in the parameter $a$, which is critical to determining of implantation profile. It is noteworthy that both the experimental data and calculations show that the positron current density to the interface can reach a fairly high value. About $75 \%$ of the implanted positrons can drift to the interface under a reverse bias greater than $25 \mathrm{~V}$. This finding indicates that semi-insulating GaAs may be a promising candidate for fabricating a highefficiency field-assisted positron moderator.

\section{ACKNOWLEDGMENTS}

The authors wish to thank B. Nielsen, Cs. Szeles, and V. J. Ghosh for many useful discussions. The help of J. P. Peng, S. Szpala, and G. Ghislotti is acknowledged. This work was supported in part by the U.S. Department of Energy under Contract No. DE-AC02-76CH-00016.
${ }^{1}$ E. Soininen, H. Huomo, P. A. Huttunen, J. Makinen, A. Vehanen, and P. Hautojärvi, Phys. Rev. B 41, 6227 (1990).

${ }^{2}$ A. P. Mills, Jr. and L. Pfeiffer, Phys. Rev. Lett. 36, 1389 (1976).

${ }^{3}$ A. P. Mills, Jr., E. M. Gulliksen, L. Pfeiffer, and W. S. Rockward, Phys. Rev. B 33, 7799 (1986).

${ }^{4}$ H. L. Au, C. C. Ling, T. C. Lee, C. D. Beling, and S. Fung, in Proceedings of the 9th International Conference on Positron Annihilation, edited by Z. Kajcsos and C. Szeles, Material Science Forum Vols. 105-111 (Trans Tech, Aedermannsdorf, 1992), p. 1363

${ }^{5}$ R. I. Simpson, M. G. Stewart, C. D. Beling, and M. Charlton, J. Phys. Condens. Matter 1, 7251 (1989).

${ }^{6}$ Y. Y. Shan, C. C. Ling, H. L. Au, S. Fung, C. D. Beling, and Y. Y. Wang, in Proceedings of the 10th International Conference on Positron Annihilation, Beijing, 1994, edited by Y. J. He, B. S. Cao, and Y. C. Jean, Material Science Forum Vols. 175-178 (Trans Tech, Aedermannsdorf, 1995), p. 517.

${ }^{7}$ P. J. Schultz and K. G. Lynn, Rev. Mod. Phys. 60, 701 (1988).

${ }^{8}$ H. Huomo, E. Soininen, and A. Vehanen, Appl. Phys. A 49, 647 (1989).

${ }^{9}$ P. J. Schultz, E. Tandberg, K. G. Lynn, B. Nielsen, T. E. Jackman, M. W. Denhoff, and G. C. Aers, Phys. Rev. Lett. 61, 187 (1988).

${ }^{10}$ B. Nielsen, K. G. Lynn, D. O. Welch, T. C. Leung, and G. W. Rubloff, Phys. Rev. B 40, 1434 (1989).

${ }^{11}$ A. P. Mills, Jr., Phys. Rev. Lett. 41, 1828 (1978).

${ }^{12}$ B. Nielsen, K. G. Lynn, A. Vehanen, and P. J. Schultz, Phys. Rev. B 32, 2296 (1985)

${ }^{13}$ H. H. Jorch, K. G. Lynn, and I. K. MacKenzie, Phys. Rev. Lett. 47, 362 (1981).

${ }^{14}$ H. H. Jorch, K. G. Lynn, and T. McMullen, Phys. Rev. B 30, 93 (1984).

${ }^{15}$ A. P. Mills, Jr. and C. A. Murray, Appl. Phys. 21, 323 (1980).

${ }^{16}$ J. Mäkinen, C. Corbel, P. Hautojärvi, A. Vehanen, and D. Mathiot, Phys. Rev. B 42, 1750 (1990).

${ }^{17}$ P. C. Rice-Evans, D. L. Smith, H. E. Evans, and G. A. Gledhill, in Positron Beams for Solids and Surfaces, edited by P. J. Schultz, G. R. Massoumi, and P. J. Simpson, AIP Conf. Proc. No. 218 (AIP, New York, 1991), p. 147.
${ }^{18}$ K. Saarinen, P. Hautojärvi, A. Vehanen, R. Krause, and G. Dlubek, Phys. Rev. B 39, 5287 (1989).

${ }^{19}$ E. Soininen, J. Mäkinen, D. Beyer, and P. Hautojärvi, Phys. Rev. B 46, 13104 (1992).

${ }^{20}$ A. van Veen, H. Schut, J. de Vries, A. R. Hakvoort, and R. M. Ijpma, in Positron Beams for Solids and Surfaces (Ref. 17), p. 171.

${ }^{21}$ K. G. Lynn, W. E. Friez, and P. J. Schultz, Phys. Rev. Lett. 52, 1137 (1984).

${ }^{22}$ D. Schodlbauer, P. Sperr, G. Kogel, and W. Triftshäuser, Nucl. Instrum. Methods Phys. Res. Sect. B 34, 258 (1988).

${ }^{23}$ D. T. Britton, J. Phys. Condens. Matter 3, 681 (1991).

${ }^{24}$ W. E. Frieze, K. G. Lynn, and D. O. Welch, Phys. Rev. B 31, 15 (1985).

${ }^{25}$ J. Mäkinen, C. Corbel, P. Hautojärvi, and D. Mathiot, Phys. Rev. B 43, 12114 (1991).

${ }^{26}$ S. Valkealahti and R. M. Nieminen, Appl. Phys. A 32, 95 (1983).

${ }^{27}$ K. G. Lynn, D. M. Chen, B. Nielsen, and R. Pareja, Phys. Rev. B 34, 12114 (1986)

${ }^{28}$ K. G. Lynn and D. O. Welch, Phys. Rev. B 22, 99 (1980).

${ }^{29}$ S. Valkealahti and R. M. Nieminen, Appl. Phys. A 35, 51 (1984).

${ }^{30}$ A. Vehanen, K. Saarinen, P. Hautojärvi, and H. Huomo, Phys. Rev. B 35, 4606 (1987).

${ }^{31}$ J. A. Baker, N. B. Chilton, and P. G. Coleman, Appl. Phys. Lett. 59, 164 (1991).

${ }^{32}$ P. Asoka-Kumar, K. G. Lynn, and D. O. Welch, J. Appl. Phys. 76, 4935 (1994).

${ }^{33}$ M. J. Puska and R. M. Nieminen, Rev. Mod. Phys. 66, 841 (1994).

${ }^{34}$ R. M. Nieminen and J. Oliva, Phys. Rev. B 22, 2226 (1980).

${ }^{35}$ J. L. Campbell, Appl. Phys. 13, 365 (1977).

${ }^{36}$ Y. Y. Shan, H. L. Au, C. C. Ling, T. C. Lee, B. K. Panda, S. Fung, C. D. Beling, Y. Y. Wang, and H. M. Weng, Appl. Phys. A 59, 259 (1994).

${ }^{37}$ S. M. Sze, Physics of Semiconductor Devices, 2nd ed. (Wiley, New York, 1981).

${ }^{38}$ C. C. Ling, T. C. Lee, S. Fung, C. D. Beling, H. Weng, J. Xu, S. Sun, and R. Han, J. Phys. Condens. Matter 6, 1133 (1994).

${ }^{39}$ Y. Y. Shan, B. K. Panda, S. Fung, and C. D. Beling, Phys. Rev. B 52, 4724 (1995). 